\title{
The Impact of Distributed Generation on the Protection of Distribution Network
}

\author{
Yang Zhao \\ Jinzhou Power Supply Branch, State Grid Liaoning \\ Electric Power Supply Co. Ltd \\ Jinzhou, china \\ Yangzhao112@163.com
}

$\mathrm{Yu} \mathrm{Li}$

Jinzhou Power Supply Branch, State Grid Liaoning

Electric Power Supply Co. Ltd

Jinzhou, china

Liyu1988@163.com

\author{
Honggang Yang \\ Jinzhou Power Supply Branch, State Grid Liaoning \\ Electric Power Supply Co. Ltd \\ Jinzhou, china \\ Honggang16@163.com
}

\begin{abstract}
Distributed power access to change the trend and fault characteristics of traditional distribution network, the existing distribution network protection caused a huge impact. To meet the distributed power of access, protection methods must examine with the grid. After the analysis of distributed power grid access with fault current characteristics and classification of different principles expounded distributed power distribution grid Protection. On the basis of the distribution network including distributed power protection configuration overview home and abroad, analysis and discussion of existing methods of protection and pointed out the direction of development of distribution network protection configuration. Further research is distributed power grid access with protection configuration to provide ideas for the ultimate realization of the future distribution network safe, reliable operation.
\end{abstract}

\section{Keywords- DG; distribution network; protection; influence}

\section{INTRODUCTION}

Distributed generation is a new power supply technology, which is a product of the rise of the Earth's environment and sustainable development policies of technological progress in the development of the second half of the 20th century, the power industry is along such a mode, power plants that generate electricity from high-voltage power transmission grid, including high, medium and low voltage power distribution system to distribute power to the user in such a system, only one trend direction: from the power plant to the grid again to the user. With access after a large number of distributed power distribution network, this pattern will change, energy production will be closer to the user. However, due to the distributed power access to the distribution system, will inevitably distribution system so complex, difficult to run and schedule[1-3].

Distributed power grid will be incorporated into the distribution network to bring a series of impact, the impact of distributed power generation on the distribution network associated with the operation of the distribution network and distributed power. Distribution network was radial structure is characterized by a single power supply, distribution network protection is as a basis for the design. When access to distributed power distribution network, distribution network structure will change When the distribution network failure, in addition to the system provides fault current to the point of failure, the distributed power failure points will also provide fault current, changing the distribution network of the shortcircuit level nodes thereby affecting the distribution grid protection devices normal operation[4]. type distributed power, installation location and capacity and other factors will affect the distribution grid protection.

Therefore, with a relay configuration problems including distributed power grid has attracted the attention of many scholars. This paper describes the impact of distributed power classification of different principles to protect the distribution network, including on the basis of distributed power distribution network protection configuration overview home and abroad, analyze and discuss the existing methods of protection, concluded that the distribution network protection Configuring direction.

\section{DISTRIBUTED GENERATION}

Distributed generation itself is not a new form of small thermal power early in our country, as well as in small thermoelectric important industry and places the user in order to enhance the reliability of power supply to install their own power supplies are all distributed power. Nevertheless, the academic definition of DG remains controversial. CIGRE Committee (CIGRE) The DG is defined as: a maximum capacity of 50-100MW, usually coupled to the power distribution network and is not unified scheduling and control of generators. According to this definition, access transmission 
system containing hundreds of large-scale wind farm wind turbine is not a column of the DG[5]. DG IEEE definition of a small capacity, the system can be in any position of power grid generators. Many definitions DG, overall based on two criteria: the capacity and grid voltage levels. The rated capacity of the DG, IEEE, EPRI and CIGRE and other international organizations have written a report on its description, but there is no consensus among the three, as defined by IEEE DG capacity range of less than $10 \mathrm{MW}$, DG capacity range defined by EPRI In between a few $\mathrm{kW}-50 \mathrm{MW}$, DG capacity range CIGRE given 50-100MW. DG consideration from the grid voltage level, internationally, including most scholars believe that DG is coupled to the power distribution system and installed near the load is coupled to the transmission system of generators[6].

Distributed generation and distribution networks main contact, has many advantages, but its impact on the entire power system is indeed enormous. First, the impact of distributed power on the hair, the transmission system is that the demand for the new centralized power plants and transmission lines will reduce the distance; secondly, the impact on the distribution system that will radically change the distribution system, namely from the original single-ended power supply radial network becomes multiport networks, protection and control and management of the distribution network will become more complicated; again, distributed power system, the electricity market will have a profound impact.

Distributed generation including fuel cells, small cogeneration and small hydroelectric power stations, wind power, solar arrays and other forms of biomass power generation. Distributed generation is usually the main access to the distribution network, would be the distribution network The impact on many aspects, such as: for power quality, grid reliability, short-circuit current protection effects and so on[7]. Distributed generation, such as wind power, solar power, adopted a lot of power electronic devices, such as inverters, their design and application design conditions than are strictly prohibited by a large current, which leads to lack of protection required to trigger a short circuit capacity. Protection from the grid perspective, the main disadvantage of this system is the lack of overload. Small and medium-capacity access to distributed power distribution network, will provide the point of failure fault current at the time of failure. From the perspective of research in terms of protection, distributed power model can be a power series reactor model to represent. Thus the need to consider is that when a fault occurs in a distributed power supply capable of providing much fault current. For different types of distributed power source, the reactance value is somewhat different, it represents the fault current of the power supply injectivity.

Production and use of electricity in the same place or restricted in the local area, to achieve economic, efficient and reliable power generation is the main characteristics of distributed power generation. DG delay due to load growth or expansion of the need for an additional power system facilities, to reduce or avoid investing in transmission and distribution networks; between the user can provide partial load, especially in the peak load times can reduce the user total electricity required to pay, and can provide emergency or back-up power for the user to improve the reliability and security of power distribution network stability. Distributed generation can be configured in the load area effectively, increase the stability of the distribution network and improve the distribution network voltage and load power factor, slow update speed of the system and increase grid reliability and economy. On the macro level, the use of distributed power generation and distribution technologies help take advantage of abundant clean and renewable energy around the strategy for the implementation of sustainable development is of great practical significance.

\section{DISTRIBUTION NETWORK PROTECTION}

The basic principles of power system protection is to identify changes in the difference between normal operation and fault systems and non-electrical quantities of electrical quantities, using a principle to identify the characteristics of these changes, the difference is more obvious representation protect better the performance. Unilateral power, the radiation power distribution network in China is currently the main power distribution system, the general configuration of instantaneous current protection, limit current protection and definite time overcurrent protection.

1) Current Protection. Current Protection refers only response current increases and can instantaneous action removal of fault protection, in order to ensure the selective action, we must ensure that the next line at the exit of the shortcircuit does not start from the protection device startup parameter tuning on, so current protection protect only part of the line.

2) limit current protection. Limit current protection refers to the time limit can be quickly removed to protect the smaller faults within the scope of the entire line, and its working principle is: to extend the scope of protection is bound to go to the next line, when the next one line at the exit of a short circuit, protection Start; selectivity of its action in order to ensure the protection it must have a certain time limit, but in order to shorten the time limit, the request does not exceed the scope of protection under the protection of a line speed off. Operating current setting principle is: starting current protection device should be in accordance with current quick escape to the next line when the end of a short circuit fault protection to the maximum short-circuit current setting[8].

3) definite time overcurrent protection. Definite time overcurrent protection refers to a protection device according to escape its starting current maximum load current setting, its role mainly as the main protection line and the next line near the reserve to protect remote backup. The working principle is: do not be action, short-circuit the normal starting time and the time to ensure that the selective action. Setting principles for action: Press escape the maximum load current of the circuit tuning; at the same time ensure that after the removal of the external fault protection device able to return.

Type distribution network topology more, including radial wiring, trunk wiring and ring net connection, in the form depends on the reliability of power supply requirements, most of the urban and rural power distribution system using radial structure. Because of this structure, reliable connection, 
protection setting easy, easy to expand and so on. Simple radial distribution network and is powered by the unilateral power structure. Our distribution network protection is as a basis for design and configuration, and low-voltage power distribution network are mostly one-sided, radial distribution network. Relay phase distribution network for high-voltage power grid in terms of protection, are simple protection. Commonly used in a current distribution network relay protection, voltage protection, over current protection and distance protection. Due to the trend of the distribution network radial flow is unidirectional, and $80-90 \%$ of failures are considered transient fault, so in order to simplify the protection configuration, typical of traditional distribution network protection design is usually the main feeder breaker using current protection and over-current protection consisting of two-stage protection, and configure the three-phase reclosing device in which current protection in accordance with the end of the line faults tuning sensitivity of the method, can not protect the line length; overcurrent protection only protects the full length of the line, and can protect the full length of the adjacent lines, can play the role of remote backup protection; while the branch line is used to protect high-voltage fuses.

\section{IMPACTS OF DG ON DISTRIBUTION SYSTEM PROTECTION}

After the distributed power distribution network access, the impact on the distribution network may be positive, it could be negative, depending on the operating characteristics of distributed power. Active role mainly in flexible, reliable on, including: increasing the available capacity distributed power grid, the valley has shifted the role of clipping; large numbers of distributed power to reduce the constant need for new largescale power plants, saving investment in building power plants and transmission and distribution equipment; distributed generation for electricity production closer to the load, reducing power transmission losses in the network; distributed generation can be run with a load islands. When the system fails, the distributed power continue to part-load power. This can reduce the power range and improve reliability.

However, there are also the following adverse effects: distributed power the load forecasting of power system planning and operation of greater uncertainty, greater difficulty to find the optimal network layout program; distributed power to change the trend of the distribution network system unidirectional flow characteristics, such that the trend can not be predicted, and also generates a series of reliability of power quality problems, affect system operation; fault current level distributed power system changes, the duration and direction, the impact protection reliability and safety ; unplanned islanding system may be, the user equipment, maintenance personnel harm.

\section{A. Impact of DG on distribution network Current Protection}

The traditional distribution network is mainly single power supply. Radial structure, this distribution network structure is simple, small investment, easy maintenance. DG access to distribution networks will change the original structure of the grid, the grid will have an impact on the short-circuit current with the distribution and protection between. When the distribution network access larger capacity or more small capacity DG, DG due to traffic diversion branch where, after a fault current flowing through the protection device may be reduced, reducing the scope of the protection protection.

DG access to the distribution network may also be at fault when an adjacent line, where the line DG reverse current due to no fault cause tripping.Line L2 contains DG. If the line L1 fails, DG provide short-circuit current protection circuit breaker QF2 fault current is detected at the fault point via the bus, if the protection setting is exceeded, action jump QF2, will lead to the power supply line L2 is interrupted As shown in Fig. 1.

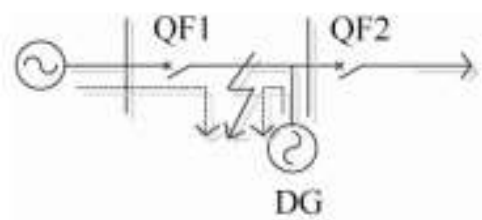

Figure 1. Impacts of DG on current protection with DG at substation

DG improve access throughout the short-circuit current level of the grid, but may reduce the individual branches of the former than the short-circuit current DG access; DG location and capacity have a significant impact on the distribution network in the distribution and size of the short-circuit current, DG's access may cause trouble loading feeder fuse blown.

\section{B. Impact of DG on distribution network automatic reclosing}

$80-90 \%$ of the distribution network part of the fault is transient. Reclosing applications to improve system reliability, reduce maintenance workload grid has a very important role. When in the radial distribution network structure, reclosing in transient fault line quickly restore power, will not have any impact on the distribution system and damage. Access DG's distribution network, after the failure, DG may still provide the fault current to the point of failure, when reclosing were coincidence, due to the power grid, and may cause fault current jump, causing reignition point of failure, resulting in insulation breakdown, and further expansion of the accident. DG access may also cause non-closing period. After the distribution network including the failure of DG, DG and its nearby islands electricity load may form, power islands is difficult to remain completely synchronized with the grid. After the power supply during this time jump to reclose when the phase angle difference between the two may appear in any one location. Asynchronous closing will make a big impact on current and transient over-voltage, the DG units and network equipment has a significant injury.

Before reclosing accelerate commonly used in multi-stage distribution lines, generally only install the appropriate reclosing device in the feeder head end, when a fault occurs at any point on the feeder protection device selectively instantaneous action, the line is disconnected, then by coincidence to be corrected. As shown in QF1, QF2 at Fig. 2. 


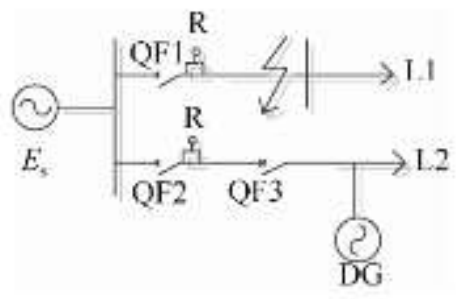

Figure 2. Impacts of DG on former acceleration reclosure

Before reclosing accelerator installed in the forefront of protecting each side of the feeder line L1 failure protection action at QF1 tripping circuit breakers, if the fault is permanent, after reclosing, by selective removal of current protection malfunction. According to the principle of setting three-current protection to protect the closer the system power supply side, the longer the settling time. Under the worst case, if a fault occurs in the place near the bus, the overcurrent protection circuit breaker QF1 may take a long time before action, if QF1 overcurrent protection for longer than breaker $\mathrm{QF} 2$ and overcurrent protection operation $\mathrm{QF} 3$ time, which resulted in QF2 and QF1 tripping QF3 prior to interrupt the normal power supply lines.

\section{DG DISTRIBUTION GRID CONTAINING PROTECTION COUNTERMEASURES}

The system can be partitioned among the regions adversely affected by the breaker DG access to the existing distribution network to protect against possible cause, by the relay distribution station to complete the online induction failure, identify the fault type and fault area and to the corresponding circuit breaker trip signal issued function, fault isolation and removal of DG region achieve fault zone system by the circuit breaker, so as to ensure the normal operation of non-fault region, and for transient faults reclosing implemented by the main relay. The method for DG when a large number of access distribution network, a wide range of the system loses its radial nature when it is effective, but for the system and there is still radiating region branch, because in a branch to the region Any equivalent impedance power supply are likely the same, this method may not work. While taking advantage of the fault current limiter to weaken the impact of DG on the grid Protection. Because the traditional fault current limiter with an additional impact on the normal operation of the power grid, as this article proposes a power electronic switch in the form of flow restrictors, which play a role in limiting device only after power failure. Through simulation analysis can draw this kind of fault current limiter can effectively suppress the short-circuit current level after DG access to the grid.

\section{CONCLUSION}

Distributed generation as a new efficient and clean power generation in the future electricity system will be widely applied. However, access to a large number of distributed power distribution network is bound to profoundly affect the structure and distribution network of the short-circuit current of the size and distribution of the flow distribution network, bringing a wide range of effects on the distribution network protection. In order to improve power distribution network reliability and power quality, analyzes the impact of distributed generation on distribution network protection and movement caused by the behavior has a certain significance to the promotion and application of distributed power generation.

\section{REFERENCES}

[1] R. Palma-Behnke, J.L.C A, L.S. Vargas, etal, “A distribution company energy acquisition market model with integration of distributed generation and load curtailment options," IEEE Transactions on Power Systems, vol. 20, pp. 1718-1727, 2005.

[2] A. Keane, E. Denny , M. O' Malley, "Quantifying the impact of connection policy on distributed generation," IEEE Transactions on Energy Conversion, vol. 22, pp.189-196,2007.

[3] T. DOYLEM, "Reviewing the Impacts of Distributed Genera2tion on Distribution System Protection ," IEEE IndustryApplicationsMagazine, , vol. 5, pp.103 -105, 2002.

[4] N. Natthaphob, T. H. Gerald, A. Raja and S. Siddharth, " Fault Current Contribution From Synchronous Machine and Inverter Based Distributed Generators. Power Delivery," IEEE Transactions on, vol.22, pp. 634-641, 2007.

[5] S. M. Brahma and A. A. Girgis, "Development of adaptive protection scheme for distribution systems with high penetration of distributed generation," IEEE Transactions on Power Delivery, vol.19, pp. 56-63, 2004.

[6] M. Pipattanasomporn, M. Willingham, S. Rahman, "Implications of onsite distributed generation for commercial industrial facilities," IEEE Trans on Power Systems, vol. 20, pp.206-212, 2005.

[7] J. C. Vieira, W. Freitas, X. Wilsun and A. Morelato, "Efficient coordination of ROCOF and frequency relays for distributed generation protection by using the application region," IEEE Transactions on Power Delivery, vol.21, pp. 1878-1884, 2006.

[8] R. C. Dugan, T. E. McDermott, "Distributed generation," IEEE Industry Applications Magazine, vol. 8, pp.19-25, 2002. 